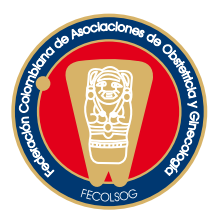

Reporte De CASO

\title{
TUMOR DE KRUKENBERG COMO MANIFESTACIÓN INICIAL DE UN CÁNCER GÁSTRICO EN UNA MUJER DE 23 AÑOS
}

\section{Krukenberg tumor as the initial manifestation of gastric cancer in a 23-year-old woman}

Jorge Alexander Rodríguez, M.D.*

Recibido: septiembre 5/2005 - Revisado: noviembre 8/2005 - Aceptado: noviembre 22/2005

\section{RESUMEN}

Los tumores metastásicos del ovario representan el 5-6\% de todos los tumores de ovario y generalmente se diagnostican en mujeres entre la cuarta y quinta década de vida, aunque pueden encontrarse en mujeres de cualquier edad. Son de mal pronóstico y es importante considerarlos dentro del diagnóstico diferencial en mujeres de cualquier edad y especialmente, en el caso del tumor de Krukenberg, cuando se trata de masas anexiales bilaterales. Se presenta el caso de una mujer de 23 años a quien se le practicó ovariosalpingectomía bilateral por masas bilaterales de ovario. La patología inicial reportó un tumor del grupo tecoma-fibroma, pero luego de realizar estudios de inmunohistoquímica en el Instituto Nacional de Cancerología (INC) de Bogotá, Colombia, fue clasificado como un tumor de Krukenberg. Posteriormente en su estudio, se detectó un adenocarcinoma gástrico primario y la paciente falleció cinco meses después del diagnóstico.

Palabras clave: tumor de Krukenberg, neoplasmas ováricos, metástasis del neoplasma.

\section{SUMMARY}

Metastatic tumors of the ovaries represent 5-6\% of all ovarian tumors and are generally diagnosed in

* Ginecoobstetra, Universidad de Antioquia, Fellow en ginecología oncológica, Instituto Nacional de Cancerología, Bogotá, Colombia. Correo electrónico: jorgea1231@hotmail.com women between the fourth and fifth decades of life, although they can be found in every age group. Their prognosis is poor and it is useful to regard them (especially the Krukenberg tumor) as differential diagnosis in women from any age group when faced with bilateral ovarian masses. This report presents a case of a 23-year-old woman who presented bilateral ovarian masses for which she underwent bilateral salpingo-oophorectomy. The initial pathology report showed a thecoma-fibroma tumor, but it was later classified as being a Krukenberg tumor after carrying out immunochemistry studies in the National Cancer Institute (NCI, Colombia). Primary gastric adenocarcinoma was then diagnosed; however, the patient died 5 months later.

Key words: Krukenberg tumor, ovarian neoplasms, neoplasm metastasis.

\section{INTRODUCCIÓN}

Los tumores metastásicos del ovario constituyen el 5-6\% aproximadamente de todos los tumores de ovario. A este grupo de neoplasias pertenece el tumor de Krukenberg, es uno de los más representativos y por lo general constituye un dilema diagnóstico, sobre todo cuando se presenta como una manifestación inicial sin que se haya descubierto aún el tumor primario. Generalmente se diagnostican en mujeres entre la cuarta y quinta década de vida; por lo tanto, cuando se encuen- 
tran masas anexiales bilaterales en una paciente joven, el diagnóstico prequirúrgico generalmente no toma en cuenta un tumor metastásico. Sin embargo, este diagnóstico diferencial debería ser tomado en cuenta cuando se enfrenta este tipo de casos. El objetivo de esta publicación es presentar el caso de una paciente de 23 años con un tumor de Krukenberg como manifestación inicial de un adenocarcinoma gástrico.

\section{CASO CLÍNICO}

Mujer de 23 años que consultó a un hospital local de segundo nivel de complejidad de atención en febrero de 2004 por sensación de masa pélvica y sangrado uterino anormal de tres meses de evolución.

Antecedentes Ginecoobstétricos: menarquia a los 11 años, ciclos: 30 × 3, fecha de la última menstruación (FUM): febrero 11/2004, G1P1V1C0A0, planificación familiar: dispositivo intrauterino.

$\mathrm{Al}$ examen físico se encontró una paciente en buenas condiciones generales, con una masa abdominal de $8 \mathrm{~cm}$ aproximadamente, ubicada en la fosa ilíaca derecha; al tacto vaginal se encontró una masa en fondo de saco derecho correspondiente al polo inferior de la masa palpada por vía abdominal, móvil, dolorosa, de aproximadamente $9 \mathrm{~cm}$, útero de $8 \mathrm{~cm}$ en anteversoflexión.

La ecografía transvaginal mostró una masa en fosa ilíaca izquierda de ecogenicidad mixta, solidoquística de $104 \mathrm{~mm}$ x $77 \mathrm{~mm}$ x $76 \mathrm{~mm}$ y en la fosa ilíaca derecha una masa de $151 \mathrm{~mm}$ x $83 \mathrm{~mm}$ solidoquística compatible con teratoma.

Se realizaron marcadores tumorales, los resultados fueron: AFP: negativo, ACE: negativo, CA125: $104 \mathrm{UI} / \mathrm{ml}$.

Con el diagnóstico de masa pélvica bilateral fue llevada a laparotomía en marzo 8/2004, encontrando masa de ovario bilateral, por lo cual se realiza ovariosalpingectomía derecha y resección parcial del ovario izquierdo. La patología informó: tumor ovárico bilateral del grupo tecoma-fibroma.

La paciente fue evaluada en el servicio de ginecología oncológica del INC en junio 11/2004 refi- riendo dolor pélvico de una semana de evolución, al examen físico se encontraron como hallazgos positivos masa pélvica de predominio central, lateralizada a la derecha, de $12 \mathrm{~cm}$; en el tacto vaginorrectal se palpó en el fondo de saco de Douglas el polo inferior de la masa descrita en el abdomen la cual es dolorosa, no se diferencia útero, cuello uterino de $3 \mathrm{~cm}$, duro y sin volumen tumoral. En el recto se encuentra masa de aproximadamente $2 \mathrm{~cm}$ de diámetro, localizada a $8 \mathrm{~cm}$ del esfínter anal externo.

El informe de la revisión de láminas histológicas en el INC fue: (junio 17/2004): adenocarcinoma metastásico de ovario bilateral con células en anillo de sello (tumor de Krukenberg), se confirma mediante inmunohistoquímica positiva para PAS, AE 1, AE 3, CK 20 y Mucicarmina.

Con el diagnóstico de Tumor de Krukenberg se realizó colonoscopia, la cual es negativa y endoscopia de vías digestivas altas la cual informó: la mucosa del cuerpo gástrico presenta pliegues engrosados e irregulares con distensión, comprometida e indurada de la cual se toman biopsias. Reporte de biopsias de endoscopia: adenocarcinoma mal diferenciado de tipo difuso. Con este diagnóstico se envía la paciente al servicio de gastroenterología para valoración y manejo prioritario en donde se realizó el diagnóstico de adenocarcinoma gástrico con metástasis bilaterales a ovario (tumor de Krukenberg). Se considera que la paciente no es candidata para manejo curativo.

La paciente presenta deterioro progresivo del estado general y fallece en casa en octubre $1 / 2004$.

\section{DISCUSIÓN}

El tumor de Krukenberg es un tumor raro, de origen metastásico cuya principal característica microscópica es la de presentar células en anillo de sello. El origen primario en el $80 \%$ de los casos es un cáncer gástrico, se presenta a una edad promedio de 45 años; el 70\% de las pacientes tienen mas de 40 años. Dentro de sus características clínicas se encuentra la presentación en 
forma bilateral en el $80 \%$ de los casos, el tamaño es de 5 a $10 \mathrm{~cm}$ y su consistencia es predominantemente sólida, además se puede encontrar en el 30\% de los casos como manifestación primaria y la localización del primario puede quedarse sin diagnóstico hasta en el $40 \%$ los casos. ${ }^{1-3}$

Existen falsos positivos en el diagnóstico histológico, entre los cuales se tienen el cistoadenocarcinoma de células claras del ovario, el carcinoide mucinoso y específicamente el tecoma-fibroma que se puede confundir con la variante tubular del tumor de Krukenberg que contiene luteinización estromal. Por ello, es necesario realizar inmunohistoquímica con marcadores como mucicarmina y PAS (Ácido Periódico de Schiff), que de ser positivos confirman el diagnóstico de tumor de Krukenberg. ${ }^{4-6}$

El pronóstico es muy pobre a pesar del tratamiento rápido con tumorectomía y localización del tumor primario, siendo la supervivencia promedio de 9 meses. ${ }^{7-9}$ El tumor de Krukenberg es inusual en frecuencia, sin embargo, el hallazgo prequirúrgico de masas bilaterales anexiales pequeñas y de predominio sólido debe alertar al clínico sobre la posibilidad de encontrarse frente a un tumor de Krukenberg en cualquier grupo etario.

El diagnóstico de esta patología requiere una evaluación anatomopatológica exhaustiva, con el fin de no dar falsas expectativas a la paciente con un diagnóstico benigno o menos agresivo que el de tumor de Krukenberg.

Agradecimientos: Dr. Joaquín Luna, y al Dr. Marco Venegas pertenecientes al grupo de ginecología oncológica y al grupo de investigación clínica del Instituto Nacional de Cancerología, respectivamente.

\section{REFERENCIAS}

1. Young RH, Scully RE. Metastastic tumors of the ovary. En: Kurman RJ, ed. Blaustein's Pathology of the female genital tract. Fourth edition. New York: Springer Verlag; 1994. p. 939-75.

2. Tavassoli FA, Devilee P (Eds). World Health Organization Clasification of tumors. Pathology and genetics of tumors of the breast and female genital organs. Lyon: IARC Press; 2003. p. 193-96.

3. Moore RG, Chung M, Granai CO, Gajewski W, Steinhoff MM. Incidence of metastasis of the ovaries from nongenital tract primary tumors. Gynecol Oncol 2004;93:87-91.

4. Petru E, Pickel H, Heydarfadai M, Lahousen M, Haas J, Schaider H, et al. Nongenital cancers metastases to the ovary. Gynecologic Oncology 1992;44:83-6.

5. Holtz F, Hart W. Krukenberg tumors of the ovary. A clinicopathologic analysis of the 27 cases. Cancer 1982;50:2438-47.

6. Vang R, Bague S, Tavassoli FA, Prat J. Signet-ring stromal of the ovary: clinicopathologic analysis and comparison with Krukenberg tumor. Int J Gynecol Pathol 2004;23:45-51.

7. Kim NK, Kim HK, Park BJ, Kim MS, Kim YI, Heo DS, et al. Risk factors for ovarian metastasis following curative resection of gastric adenocarcinoma. Cancer 1999; 7:1490-9.

8. Kim HK, Heo DS, Bang YJ, Kim YI, et al. Prognostic factors of Krukenberg's tumor. Gynecol Oncol 2001;82:105-9.

9. Cheong JH, Hyung WJ, Chen J, Kim J, Choi SH, Noh SH. Survival benefit of metastasectomy for Krukenberg tumors from gastric cancer. Gynecol Oncol 2004;94:477-82. 